\title{
Weaving life's pattern
}

\section{Melvin Konner}

$\mathrm{P}$ ychologists like to stress that what happens in early life - what zoologists call the juvenile phase - is not just growth, but development. The implication is twofold. First, 'growth' suggests mere augmentation, either through increasing cell size (hypertrophy) or successive mitotic divisions (hyperplasia). But a system such as the brain could not emerge so simply. Second, 'growth' implies an autonomous process, governed from within, and (given minimal input such as oxygen and nutrients) under fairly tight genetic control. An alternative term, maturation, suggests that the transformations of early life transcend hypertrophy and hyperplasia, yet still follow a preset programme. But this neglects to consider the environment's shaping role, which, in the nervous system at least, includes learning.

So development is not just more than growth - it is more than maturation, requiring constant negotiation with the environment. Sometimes this truth has led to a refusal to try to tease out the different roles of maturation and learning. In Jean Piaget's theory of mental development, for example, the contributions of learning and of a tacitly assumed preset programme are deliberately obscured. In another model, really a metaphor, maturation and learning are viewed as the warp and woof - one blue, one yellow - that give a swatch of cloth a green colour. The claim is that attempting to separate the two contributions destroys the unique product of their interaction.

Of course, a thicker, denser blue warp makes the cloth a bluer green. These features

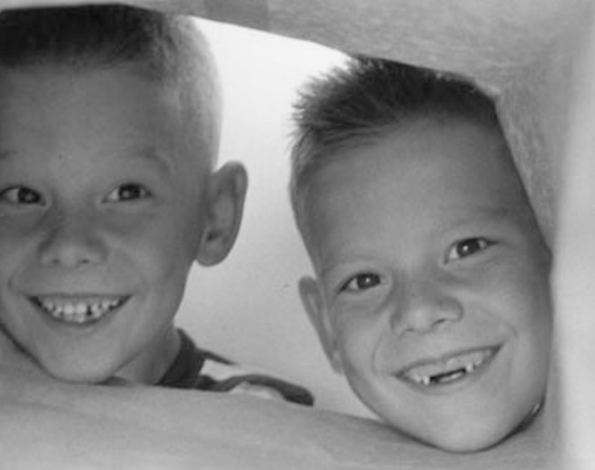

Two of a kind? From before birth, chance and the environment conspire to make twins differ. of the warp, not to mention the design and technique of weaving, help to explain the outcome. In the 1950s, the prescient psychologist Anne Anastasi saw that the real question is not "which?" or "how much?", but "how?" Advances in genetics and brain science now leave us in no doubt that we can answer all three. But how do we address the "how" question?

In embryology, development always entails interaction, although the interactions often take place inside the organism. In the classic account, the dimpling of the vertebrate eye from a blob-like to a cup-like shape - the formation of the retina - occurs in response to a chemical signal from the overlying ectoderm. Soon after, the lens is formed from ectoderm when the brand-new eye cup sends back another signal.

Later, as neurons form and migrate around the brain, they are attracted, rerouted and stopped by molecular signals. Many of these come from other cells that guide or challenge the migrators in a kind of immunochemistry. Recognition of cells and surfaces, and ultimately adhesion to them, determine the fates of neurons, and subsequently those of their extensions. These patterns become the wiring plan of the brain.

But this line of thinking comes up against Changeux's paradox: how do 30,000 human genes determine $10^{11}$ cells with $10^{15}$ connections? Obviously they can't do it in the same way that the roundworm's 18,000 genes govern its 959 cells. There are several solutions.

First, pioneer cells and axons pave the way for thousands or tens of thousands of others to track their guidance, offering lots of hook-ups for the price of one. Second, the mammalian brain forms many more cells and connections than it needs, subsequently pruning back around half of them. Some of this occurs through programmed cell death, but much depends on activity - meaning that spontaneous and reactive fetal movements shape the brain. Third, small groups of neurons may form under strict genetic control - creating small, deterministically wired systems similar to the roundworm's brain - and then compete for incoming stimulation and outgoing actions.

These processes have been called darwinian, but this is only a partial analogy. The cells of the embryo are genetically identical, and they produce no offspring, thus undermining two pillars of Darwin's theory variation and inheritance. Still, the processes involve competition, which is resolved by environmental, adaptive selection. And the cells are not quite genetically identical — the same set of genes is always there, but only

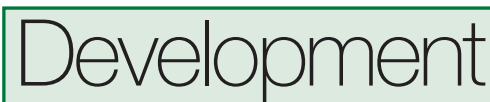

Development is not just more

than growth - it is more than

maturation, requiring constant

negotiation with the environment.

some are switched on. Which switch on and which off in any given cell - and when, and how, and why - determine the cell's character and function. A main key to development is this on-off pattern, a pulsing, embryo-wide light show that turns genetic instructions into animals.

Elucidating the control of these switches - by signals inside the cell, beyond it, or even outside the body - is the main task of biology in the twenty-first century. And the switches are not flipped just in early life - genes that confer Huntington's and Alzheimer's diseases are switched on decades after the die is cast. But of course, in a complex animal, much is left to chance. Chaos in the formal sense - exquisite sensitivity to variations in starting conditions - cumulatively amplifies small differences. This embryonic butterfly effect gives identical twins different brains within weeks of conception. Such unpredictable paths help to explain why twins differ before we even consider their environmental influences.

Less certain is the role of emergence in development, but if self-organizing processes occur in non-living solutions, why not in a minuscule protoplasmic pool or an early, inchoate blob of cells? In computer models of embryos, self-organization looks to be adequate for certain tasks. We need to learn more about these less deterministic routes to life's complexity.

One thing is certain. The sequencing of the genome will soon look like the easiest thing that biologists ever did. And what sequencers euphemistically call "annotation" and the rest of us call development what the genes actually do - constitutes the real code of living systems. To crack that code will take centuries, but getting there will be more than half the fun.

Melvin Konner teaches at Emory University, Atlanta, Georgia, USA. He is the author of the completely revised edition of The Tangled Wing: Biological Constraints on the Human Spirit.

\section{FURTHER READING}

Anastasi, A. Psychol. Rev. 65, 197-208 (1958). Changeux, J.-P. Neuronal Man: The Biology of Mind (trans. Garey, L.; Princeton Univ. Press, 1997). Edelman, G. M. Neural Darwinism: The Theory of Neuronal Group Selection (Basic, New York, 1987). Wolpert, L. The Triumph of the Embryo (Oxford Univ. Press, 1991). 